\title{
Final Report: Novel nanowires as probes of electron coherence and correlations in restricted geometries (DE-FG03-01ER45946)
}

May 17, 2005

\author{
D. Natelson \\ (Rice University, Department of Physics and Astronomy)
}

\begin{abstract}
This is a final summary report of the research conducted under DE-FG03-01ER45946, which was a research program using metal nanostructures to examine quantum coherence of electrons in normal and ferromagnetic metals. This program was the PI's first federal research grant, and by augmenting with other funds (Packard Foundation), this grant supported two graduate students during its duration. In normal metal nanostructures, quantum coherence was assessed by two independent techniques: weak localization magnetoresistance, and time-dependent universal conductance fuctuations (TDUCF noise). This work found that, in AuPd nanowires, the coherence information inferred from these two techniques were quantitatively consistent, even in the presence of magnetic impurity and phonon scattering. This confirmed theoretical expectations. However, in $\mathrm{Ag}$ and $\mathrm{Au}$ wires, the two techniques disagree, with noise measurements indicating a lower coherence length at low temperatures than weak localization. We have a candidate explanation for this, and are finishing these experiments. This work shows that subtleties remain in our understanding of coherence processes even in normal metals, particularly those involving the tunneling two-level systems that produce low frequency noise; this has relevance for quantum information processing implementations using metal devices. We have also studied time-dependent universal conductance fluctuations in ferromagnetic metals for the first time. The TDUCF in ferromagnetic nanowires show that the Cooperon channel of coherent processes is suppressed in these correlated materials. Furthermore, the surprisingly steep temperature dependence of the noise suggests that decoherence in these systems is through a different process than in normal metals. We are finishing measurements of "magnetofingerprint" conductance fluctuations in ferromagnetic metals to examine this unusual temperature dependence with an independent technique. This program has produced three papers (one Plyys. Rev. B Rapid Communication, one PRB Brief Report, and a longer PRB article), with two more in preparation; it has also resulted in six APS contributed talks by students, and two invited seminars by the PI.
\end{abstract}




\section{DISCLAIMER}

This report was prepared as an account of work sponsored by an agency of the United States Government. Neither the United States Government nor any agency Thereof, nor any of their employees, makes any warranty, express or implied, or assumes any legal liability or responsibility for the accuracy, completeness, or usefulness of any information, apparatus, product, or process disclosed, or represents that its use would not infringe privately owned rights. Reference herein to any specific commercial product, process, or service by trade name, trademark, manufacturer, or otherwise does not necessarily constitute or imply its endorsement, recommendation, or favoring by the United States Government or any agency thereof. The views and opinions of authors expressed herein do not necessarily state or reflect those of the United States Government or any agency thereof. 


\section{DISCLAIMER}

Portions of this document may be illegible in electronic image products. Images are produced from the best available original document. 
Over the last twenty years, tremendous progress has been made in understanding the role of quantum coherence in the dynamics of electrons in solids. Because the experimental consequences of quantum coherence are most readily detected in mesoscale systems, this discipline has come to be known as "mesoscopics". The improved understanding of the role of quantum coherence in solid state systems has been driven by the development and widespread availability of sophisticated patterning and fabrication techniques with submicron resolution.

Here I summarize the results of our research efforts using state-of-the-art nanostructures as tools to address fundamental, unresolved issues related to quantum coherence of electrons in conducting systems. The background and common methods of probing coherence physics are explained below in Sect. 1. Our results from this funded research are summarized in Sect. 2. We include a bulleted list of the talks and papers, and conclude the discussion in Sect. 4.

\section{Introduction and background}

\subsection{Coherence physics in solids}

Understanding the properties of an electron in a single hydrogen atom requires quantum mechanics. However, a semiclassical model of electrons that ignores quantum interference effects and the uncertainty principle is remarkably effective at describing the properties of electrons in, e.g., copper electrical wiring. Quantum corrections to this semiclassical conduction are usually negligible in macroscopic systems at room temperature because of decoherence, the suppression of quantum interference contributions to electron probability distributions by inelastic coupling of the electrons to environmental degrees of freedom.

A precise treatment of decoherence is mathematically subtle, but a simplified discussion gives a flavor of the relevant physics. Consider adding a single electron to a typical metal. The wavefunction of this electron may be approximated as a Bloch wave with a well-defined phase, $\phi$. As this electron propagates in the metal it may scatter (diffract) elastically off static disorder (e.g. grain boundaries, crystallographic defects) while this phase evolves via the Hamiltonian. When the electron undergoes an inelastic interaction with a dynamic, environmental degree of freedom (another electron; a phonon; a magnetic impurity), its phase is altered. The coherence time, $\tau_{\phi}$, is the characteristic time-scale over which the electron's phase becomes uncorrelated with its initial phase, and quantum interference terms are therefore suppressed. The coherence length, $L_{\phi}$, is the corresponding distance traversed by an electron during this time. In a diffusive system (size $L$ larger than electronic elastic mean free path $\ell$ ) with electron diffusion constant $D, L_{\phi} \equiv \sqrt{D \tau_{\phi}}$.

Decoherence depends strongly on temperature because many of the inelastic processes responsible are driven by thermal energy (e.g. the energy contained in the thermal phonons of a material). A typical coherence length in a thin metal film at room temperature is $1-2 \mathrm{~nm}$, while $L_{\phi} \sim 1 \mu \mathrm{m}$ in $\mathrm{Ag}$ at $1 \mathrm{~K}$. At such low temperatures the dominant decoherence process in pure metals is expected to be small angle electron-electron scattering. Perturbative calculations[1] predict $\tau_{\phi} \rightarrow \infty$ as $T \rightarrow 0$, with a particular power law for the divergence that depends on dimensionality (determined by comparing $L_{\phi}$ with $L, w$, and $t$, characteristic sample length, width, and thickness, respectively).

Quantum coherence must be inferred from its effects on electronic properties. There are several quantum interference corrections to the conductance that may be used to infer $L_{\phi}$. These include (see Fig. 1):

Aharonov-Bohm / Altshuler-Aronov-Spivak oscillations. When $L_{\phi}$ is comparable to half its circumference, $L / 2$, a quasi-1d ring has a conductance correction that is oscillatory in the magnetic 


\section{0}

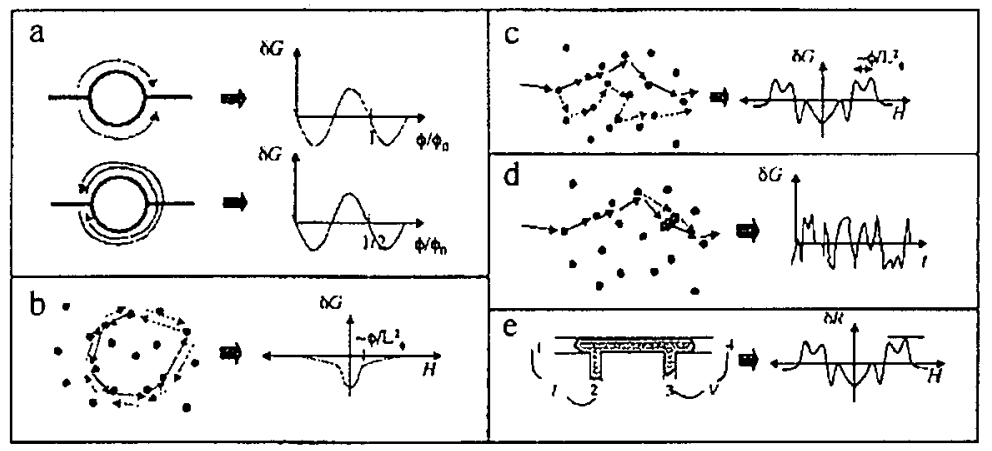

Figure 1: Various quantum interference corrections to semiclassical electronic conduction. (a) AharonovBohm and Altshuler-Aronov-Spivak oscillations; (b) Weak localization; (c) Magnetofingerprint universal conductance fluctuations; (d) Time-dependent universal conductance fluctuations; and (e) Nonlocal resistance fluctuations.

flux through the ring with a period of $h / e$. This correction is caused by the relative Aharonov-Bohm $(A B)$ phase acquired by electrons passing through the two "arms" of the ring. The amplitude of this correction is predicted[2] to vary as $e^{-L / L_{\phi}}$, allowing one to infer $L_{\phi}(T)$ from a series of magnetoresistance measurements. These oscillations average to zero in ensembles of rings.

When $L_{\phi} \sim L$, an additional oscillatory component periodic in flux like $h / 2 e$ appears. This contribution was identified[3] by Altshuler, Aronov, and Spivak (AAS) as originating from timereversed conjugate pairs of trajectories that circumnavigate the ring. When time-reversal symmetry is good (low magnetic fields), the conjugate pairs interfere constructively. The dependence of the AAS oscillations on $L_{\phi}$ continues to be a subject of interest[4]. These oscillations do not average to zero in ensembles of rings.

Weak localization magnetoresistance (WLMR)[5]. Analogous to AAS oscillations, pairs of time-reversed loop trajectories in a diffusive metal interfere constructively in zero magnetic field. For weak (strong) spin-orbit scattering, this interference enhances (reduces) the probability of electronic back-scattering, decreasing (increasing) the sample's conductance. As the magnetic field is increased, the $\mathrm{AB}$ phase eliminates this constructive interference, leading to a magnetoresistance. The characteristic field scale threads $h / e$ of flux through a typical coherent loop. Theoretical expressions allow $L_{\phi}(T)$ to be inferred from WLMR data. The WLMR does not average to zero in large samples, making it one of the easier effects to measure. The observed temperature dependence of $L_{\phi}(T)$ inferred from WLMR has been the subject of much controversy[6, 7,8$]$, particularly regarding the role of dilute magnetic impurities[9].

"Magnetofingerprint" universal conductance fluctuations (MFUCF) $(10,11]$. Conductance in mesoscale systems is the result of the interference of all possible electronic trajectories through the sample. The $\mathrm{AB}$ phase alters the relative phases of these complicated paths, leading to samplespecific, reproducible conductance fluctuations within a coherent volume. The typical correlation field is again set by the flux through a typical coherent loop. Within a coherent volume and ignoring thermal smearing, the typical conductance fluctuation is $\delta G_{\mathrm{rms}} \sim e^{2} / h$, independent of the details of the sample. The quantitative details of the MFUCF have also been the subject of controversy $[12,13]$.

Time-dependent UCF (TDUCF). Motion of the scattering sites in the material also shifts relative 


\section{○}

phases and can lead to UCF $[14,15]$. For typical fluctuators (two-level systems, or TLS) in disordered materials[16], the result is $1 / f$ distributed conductance noise. Since the fluctuations are suppressed by ensemble averaging, this noise grows as $T \rightarrow 0$, through increases in $L_{\phi}$ and reduced thermal smearing. If the number of active fluctuators, $n(T)$, is known, it is possible to infer $L_{\phi}(T)$ from the temperature dependence of the noise power, $S_{G}(B, T)$. The dependence of $S_{G}(B, T)$ on magnetic field is more typically used to extract $L_{\phi}(T)$. The component of TDUCF due to time-reversed paths is suppressed, again on a field scale that may be related to $L_{\phi}$. The TDUCF can be saturated or unsaturated. In the saturated limit, the typical size of a fluctuation within a coherence volume is comparable to the size of the MFUCF. In the unsaturated limit, fluctuations as a function of time are significantly smaller than those as a function of field. Whether a particular material is in the saturated limit depends on the size $L_{\phi}$ and the microscopic properties of the TLS that produce the noise. Unsaturated and saturated noise have different forms of magnetic field dependence. Simple consistency checks imply that most experiments have been well in the unsaturated regime.

Nonlocal resistance effects. In nanostructures where the distance between all current and voltage probes is comparable to $L_{\phi}$, nonlocal resistance effects are observable[17]. For example, see Fig. 1d. Within a coherent volume, voltage "magnetofingerprint" fluctuations would be expected between leads 3 and 4 even though classically the current would be expected to flow only from 1 to 2 . The amplitude of these nonlocal effects is expected $[18,19]$ to decay like $e^{-s / L_{\phi}}$ with the separation, $s>L_{\phi}$, between the voltage probes and current path.

Observing and characterizing these phenomena in normal metals and semiconductor devices created the field of "mesoscopic physics" in the 1980s and early 1990s.

\subsection{Coherence in ferromagnets}

The interplay between electronic correlations and quantum coherence is subtle. In the superconducting state, quantum coherence over macroscopic distances is well known. Ferromagnetic (FM) systems are another common class of electronically ordered materials, but quantum coherence in ferromagnetic metals remains comparatively unexplored.

Quantum corrections to conduction in FM systems are of fundamental interest due to correlationinduced degrees of freedom not present in normal metals (e.g. spin waves), and the interplay of FM order with coherence. These corrections have been discussed while considering[20] domain walls effects on conduction in FM nanowires[21], and magnetoresistive effects[22] in thin FM films[23]. The conductance of a mesoscopic ferromagnet is expected[20] to be highly sensitive to domain wall motion, just as the conductance of a mesoscale normal metal is sensitive to the motion of an individual elastic scatterer[24, 25]. Domain wall motion can thus lead to UCF $[10,11]$. Further nontrivial coherence effects proposed in FMs include Berry's phase physics due to coherent diffusion of spins through nonuniform magnetization[26], and dephasing via domain wall[20,26,27] and spin wave[28] scattering.

No systematic experimental examination has been reported concerning electronic coherence in FM materials and the effects of domains. The two most popular methods of inferring $L_{\phi}$, weak localization and MFUCF, rely on magnetoresistive measurements. In FM metals the anisotropic magnetoresistance (AMR), a bandstructure effect, significantly complicates efforts to observe WL and MFUCF. Examinations of mesoscale magnetic structures hint at UCF[29], and AharanovBohm (AB) oscillations have been observed in multidomain and single-domain mesoscopic NiFe rings $[30,31]$ at $\mathrm{mK}$ temperatures. 


\subsection{Unanswered questions}

Several issues remain unresolved regarding electronic quantum coherence effects in solids:

Do the different techniques for inferring $L_{\phi}$ agree with one another? This is a subtle issue for several reasons. Decoherence by small-angle electron-electron scattering is not a simple decay with a single, well-defined rate[32], leaving some ambiguity about choosing a characteristic $t_{a} u_{\phi}$. Other processes with other characteristic times are also important in these systems, including momentum relaxation (the out-scattering time in the semiclassical Boltzmann equation), spin-flip scattering, and spin-orbit scattering. Many subtleties exist in the interplay between these processes and the coherence effects outlined above.

Do we quantitatively understand the relevant decoherence processes? Most of the community now agrees that apparent saturation[6] of $L_{\phi}(T)$ as $T \rightarrow 0$ in normal metals is often due to extrinsic effects $[7,9]$ such as dilute magnetic impurities. Precise measurements and comparisons of the various coherence corrections can further constrain and resolve such discussions[33, 13].

What is the microscopic nature of the fluctuators that cause TDUCF, and can they be controlled? The microscopic TLS in metals are not known, though likely candidates include atoms at grain boundaries and mobile dislocations[16]. TDUCF are a sensitive probe of those fluctuators, and novel nanostructures and surface treatments provide ample opportunities for study.

What are the dominant decoherence processes in ferromagnetic metals? This issue is of fundamental interest and potentially of significant technological relevance, and is largely unexplored at present.

\section{Results of this program}

Since May, 2002, I have had two graduate students pursuing research on these topics with DOE support, one (Aaron Trionf) primarily examining normal metals and the other (Sungbae Lee) ferromagnetic structures. Initial investigations were complicated by dilution refrigerator problems (manufacturer's defects) that lasted several months. Despite these challenges we made significant progress in addressing some of the questions above.

\subsection{Normal metals: noise and weak localization}

We have performed precise measurements of $L_{\phi}^{\text {WL }}$ and $L_{\phi}^{\text {TDUCF }}$ on quasi-1d and quasi-2d AuPd wires down to $2 \mathrm{~K}$. Previous experiments on quasi-2d Ag films $[37,36]$ found disagreement between the lengths inferred from the two techniques at temperatures such that $L_{\phi}>L_{\mathrm{SO}}$, the spin-orbit length. These data were interpreted as confirming a theoretical expectation[38] that the relevant coherence length in UCF is the out-scattering length, rather than the inelastic length relevant in WL. This theory has been superceded[39], and the theoretical prediction is that $L_{\phi}^{\text {WLMR }}$ and $L_{\phi}^{\text {TDUCF }}$ are expected to agree precisely, as long as the only source of small-energy-transfer scattering is small-angle electron-electron (Nyquist) scattering.

We performed the first comparison of these two lengths in the presence of strong spin-orbit scattering ( $L_{\mathrm{SO}}<\sim 10 \mathrm{~nm}$ in AuPd) and dilute magnetic impurities. We found excellent agreement between $L_{\phi}^{\text {WLMR }}$ and $L_{\phi}^{\text {TDUCF }}$ over the whole range of dimensionalities and temperatures, as shown in Fig. 2a. This agreement exists despite the presence of other scattering mechanisms, including phonons and magnetic impurities, and despite extremely strong spin-orbit scattering. 

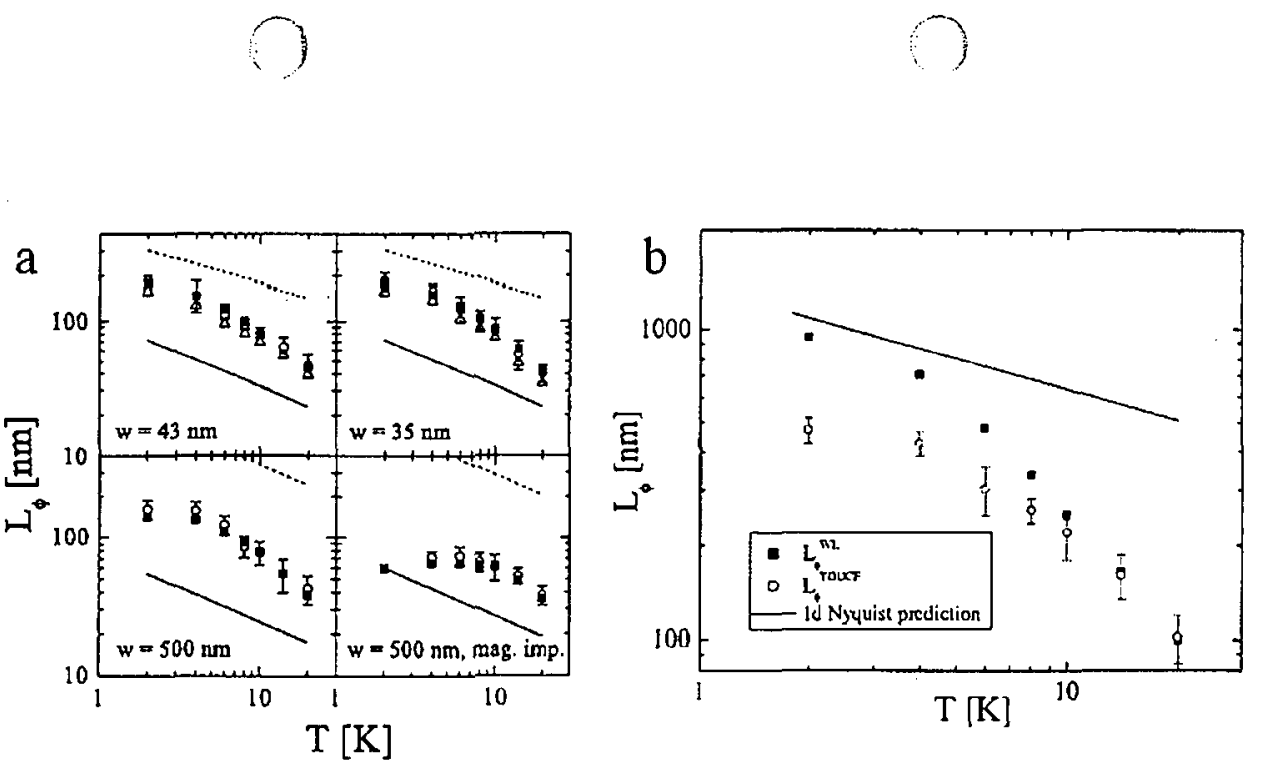

Figure 2: (a) Coherence lengths in several AuPd wires as a function of temperature, as inferred from weak localization (solid) and time-dependent UCF noise (open). The numerical consistency of the two techniques is excellent|34]. (b) Comparison of colerence lengths in a quasi-1d Ag nanowire ( $w=120 \mathrm{~nm}, L=20 \mu \mathrm{m}$, $t=12 \mathrm{~nm}$ ), as inferred from weak localization (solid) and time-dependent UCF noise (open)[35]. Note the disagreement below $\sim 8 \mathrm{~K}$, as seen previously in Ref. [36]. This inconsistency remains unexplained.

We have also examined Ag quasi-Id nanostructures[35], in an effort to understand the observations of Birge et al. mentioned above. As shown in Fig. 2b, we confirm the disagreement of these two lengths in this material, despite outstanding consistency in this system between $L_{\phi}^{\mathrm{WLMR}}(T)$ and theory, with no adjustable parameters.

This surprising discrepancy is interesting, and we have thoroughly examined this issue. Our AuPd data appears inconsistent with an explanation suggested[39] that involves triplet-channel interactions and strong spin-orbit scattering. We suggest another possible resolution to this discrepancy between the AuPd and $\mathrm{Ag}$ data: the applicability of the noise crossover expressions used in analyzing the data. Due to microscopic differences in the (unknown) fluctuators responsible for the TDUCF noise, the AuPd and Ag samples may be in different regimes. In particular, if the $\mathrm{Ag}$ samples were transitioning into the saturated noise limit, then a different form for the fitting function for noise power field dependence would be required. Fig. 3 shows the results of trying to infer $L_{\phi}^{\mathrm{TDUCF}}(T)$ using the crossover function appropriate for saturated TDUCF, in comparison with the weak localization data and the $L_{\phi}^{\text {TDUCF}}$ values calculated using the unsaturated TDUCF expression. Clearly the system is not in the saturated regime over the observed temperature range. However, the saturated crossover function data becomes a better match to the WL data as $T$ decreases. It seems reasonable that an interpolating crossover function between the saturated and unsaturated crossover functions could be necessary. Further experiments have shown the same effect in Au wires, and are ongoing.

We have also examined the drive dependence of the noise power, and found that the suppressed noise power seen at high drives is qualitatively consistent with energy averaging due to the voltage drop across a coherence length. As in the Birge experiments, we find that the coherence length itself (inferred from the field dependence of the noise) is comparatively unaffected over the range of drive currents required to suppress the TDUCF amplitude.

An initial paper on this work has been published as a Rapid Communication in Phys. Rev. $B$ [34], and was an AIP Virtual Journal of Nanoscale Physics selection. A longer Phys. Rev. B paper on the noise discrepancy in $\mathrm{Ag}$ and the drive dependence of the TDUCF has been accepted[35]. 


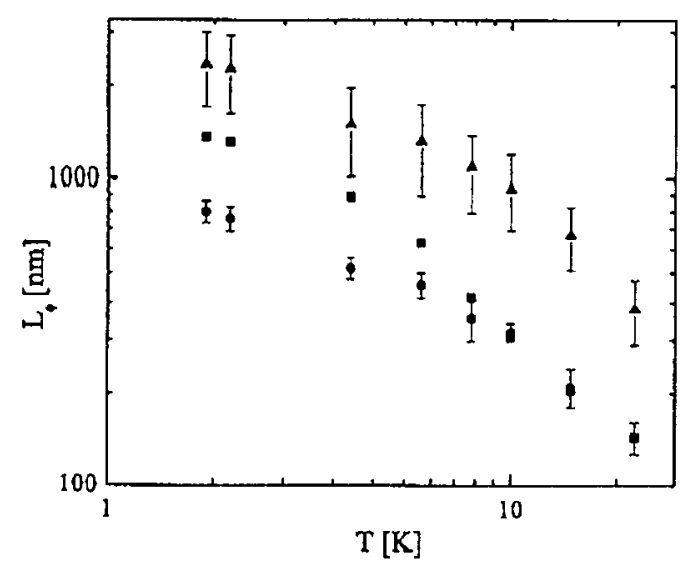

Figure 3: Coherence lengths from sample F. Squares are from WL, circles are inferred via the unsaturated TDUCF crossover function, and triangles are calculated with the saturated TDUCF crossover function.

Another paper is in preparation regarding the unexpected dependence of the coherence length and WL/TDUCF discrepancy in Au and Ag during annealing. This work has also been the topic of three March APS contributed talks, and condensed matter seminars at Columbia University and the Georgia Institute of Technology.

\subsection{Ferromagnetic metals: first studies}

The work on normal metals has served as a springboard for our studies of coherence in ferromagnetic nanowires. Using permalloy $\left(\mathrm{Py} \equiv \mathrm{Ni}_{0.8} \mathrm{Fe}_{0.2}\right.$ ) nanowires, we have performed the first TDUCF measurements of a FM metal. Since permalloy is a soft ferromagnet, shape anisotropy has been used to produce nanowire structures that exhibit either single-domain or multiple domain configurations, depending on sample geometry and field history. Fig. 4a shows magnetic force microscopy (MFM) images of segments of two such samples.

We found that the field-dependent suppression of the TDUCF used to find $L_{\phi}^{\text {TDUCF }}$ in normal metals is absent in FM nanowires as narrow as $27 \mathrm{~nm}$, as is any sign of weak localization. Given the presence of TDUCF, this suggests that while coherence persists in the FM, the cooperon contribution is suppressed. Furthermore, additional TDUCF was observed when domain walls were present and moving, providing the first experimental evidence of coherent scattering of electrons by domain walls.

Finally, the temperature dependence of the TDUCF noise power in the FM nanowires is anomalously steep when compared to that in normal metals; see Fig. 4b. There are two possibilities that would explain this, and further work (in progress) needs to be done to discern between the two. One possibility is that the fuctuators at work in the FM nanowires have a significantly different energy spectrum (though an identical relaxation rate spectrum) than those in normal metals. We believe this is unlikely, given the ubiquity of TLS fluctuators, and the fact that the TDUCF in the FM material scale with volume just as in normal metal nanowires. The other possibility is that the fluctuators in the FM are traditional TLS, in which case the anomalous temperature dependence of the TDUCF indicate an unusual $L_{\phi}(T)$. Further experiments measuring MFUCF in permalloy nanowires are underway to examine the temperature-dependent coherence processes in FM directly. 


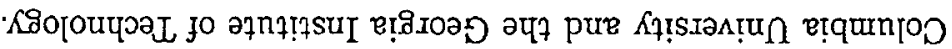

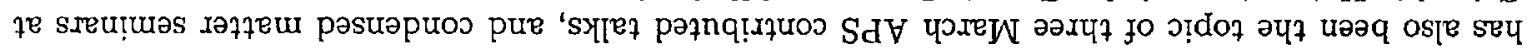

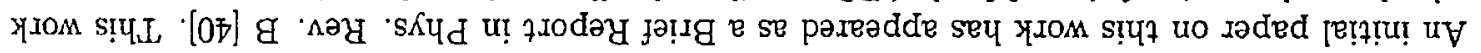

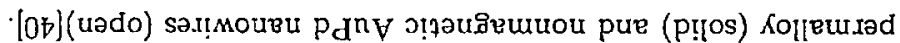

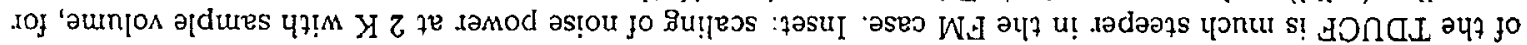

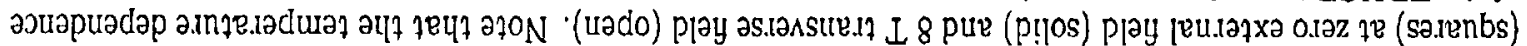

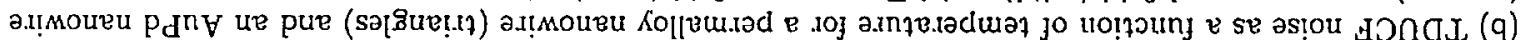

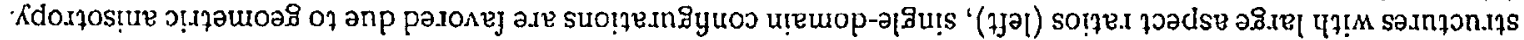

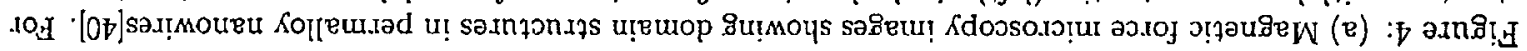
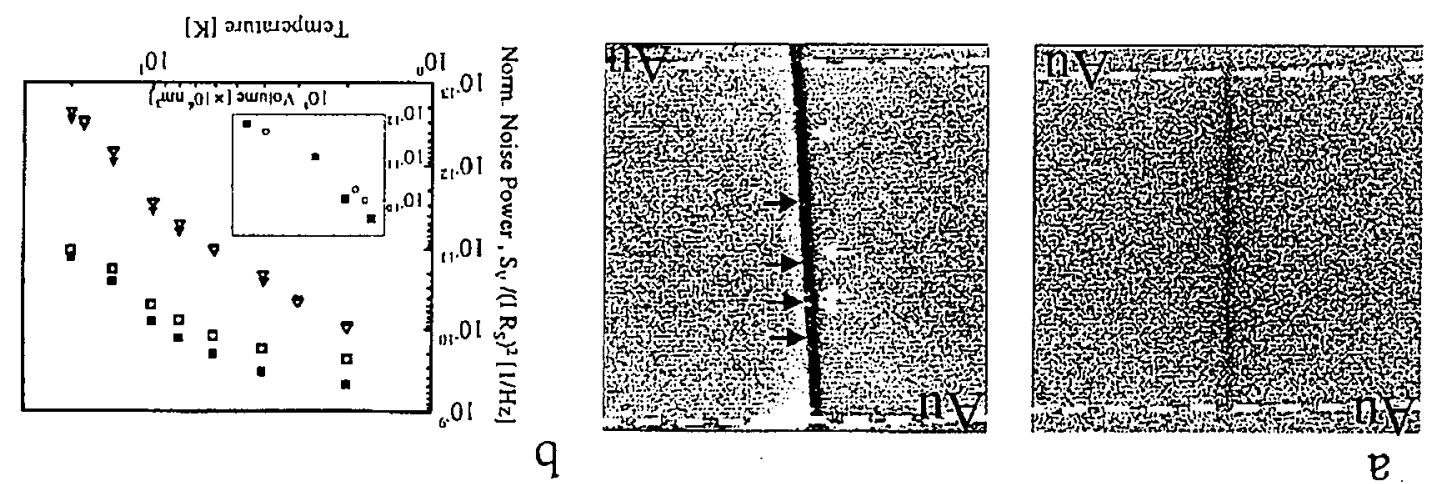

$\mathrm{q}$

$\mathcal{B}$ 


\section{Summary of accomplishments}

Three papers have resulted from this program:

- A. Trionf, S. Lee, and D. Natelson. "Electronic coherence in metals: Comparing weak localization and time-dependent conductance fluctuations", Phys. Rev. B 70, 041304(R) (2004).

- S. Lee, A. Trionfi, and D. Natelson. "Quantum coherence in a ferromagnetic metal: timedependent conductance fluctuations", Phys. Rev. B 70, 212407 (2004).

- A. Trionfi, S. Lee, and D. Natelson. "Time-dependent universal conductance fluctuations and coherence in AuPd and Ag", Phys. Rev. B (accepted for publication).

Two more papers are in preparation:

- A. Trionf, S. Lee, and D. Natelson. "Two-level systems and the effects of annealing on coherence in $\mathrm{Au}$ and $\mathrm{Ag}$ nanowires".

- S. Lee, A. Trionfi, and D. Natelson. "Magnetofingerprints in ferromagnetic nanowires".

These data have been presented at six contributed talks at APS March meetings (three on normal metals by Trionfi in 2003, 2004, and 2005; three on ferromagnetic metals by Lee in the same years). These data have also been presented by the PI in two condensed matter seminars:

- (November, 2003) Columbia University condensed matter seminar, "Coherence in solids: Kondo physics in single-molecule transistors, and coherence measurements in metal nanostructures".

- (June, 2004) Georgia Institute of Technology condensed matter seminar, "Electronic coherence in normal and ferromagnetic metals". 


\section{o}

\section{Conclusions}

This program has made significant contributions to our understanding of quantum coherence processes of electrons in metals. This is a comparatively mature field, so that the experiments and theory have advanced to the point where precise quantitative experiments are essential. Far from being "done", this field has ramifications for both fundamental science and quantum information processing. Particularly relevant for the latter is understanding what role, if any, is played by twolevel systems in decohering electrons. Careful studies of noise properties and quantum coherence effects such as the ones covered in this program are essential to a better knowledge of such systems.

We find that subtleties and mysteries remain in normal metals: what is the origin of the disagreement between coherence lengths inferred from noise and from weak localization in $\mathrm{Ag}$ and $\mathrm{Au}$, since these two techniques ostensibly measure the same physics? Why do the coherence properties of Au nanowires depend strongly on annealing?

Similarly, our results on conductance fluctation noise in ferromagnets have revealed a surprising temperature dependence. Quantum coherence effects in correlated metals are only beginning to be examined experimentally, and these data are a tantalizing hint that processes in such systems are not identical to those in normal metals.

I feel strongly enough about these issues to continue, with other funding (Packard Foundation), these investigations until they reach a natural stopping point. I regret that this area is no longer one which is in step with the programmatic needs of DOE. The publications currently in preparation and any further ones that result directly from this program will duly acknowledge DOE support with the appropriate award number. 


\section{References}

[1] B. L. Altshuler and A. G. Aronov, "Electron-electron interactions in disordered conductors," in A. L. Efros and M. Pollak, eds., "Electron-electron interactions in disordered systems," Modern Problems in Condensed Matter Physics Vol. 10, pages 1-154 (North Holland, New York, 1985).

[2] A.G. Aronov and Yu. V. Sharvin, "Magnetic flux effects in disordered conductors," Rev. Mod. Phys. 59, 755-779 (1987).

[3] B.L. Altshuler, A.G. Aronov, B.Z. Spivak, D. Yu. Sharvin, and Yu. V. Sharvin, "The AharonovBohm effect observation in metallic cylinders," JETP Lett. 35, 588-590 (1982).

[4] T. Ludwig and A.D. Mirlin, "Interaction-induced dephasing of Aharonov-Bohm oscillations," Phys. Rev. B 69, 193306 (2004).

[5] G. Bergmann, "Weak localization in thin films - a time-of-flight experiment with conduction electrons," Phys. Rep. 107, 1-79 (1984).

[6] P. Mohanty, E.M.Q. Jariwala, and R.A. Webb, "Intrinsic decoherence in mesoscopic systems," Phys. Rev. Lett. 78, 3366-3369 (1997).

[7] D. Natelson, R.L. Willett, K.W. West, and L.N. Pfeiffer, "Geometry-dependent dephasing in small metallic wires," Phys. Rev. Lett. 86, 1821-1825 (2001).

[8] A.B. Gougam, F. Pierre, H. Pothier, D. Esteve, and N.O. Birge, "Comparison of energy and phase relaxation in metallic wires," J. Low Temp. Phys. 118, 447-456 (2000).

[9] F. Pierre, A.B. Gougam, A. Anthore, H. Pothier, D. Esteve, and N.O. Birge, "Dephasing of electrons in mesoscopic metal wires," Phys. Rev. B 68, 085413 (2003).

[10] P.A. Lee and A.D. Stone, "Universal conductance fluctuations in metals," Phys. Rev. Lett. 55, 1622-1625 (1985).

[11] P.A. Lee, A.D. Stone, and H. Fukuyama, "Universal conductance fluctuations in metals - effects of finite temperature, interactions, and magnetic field," Phys. Rev. B 35, 1039-1070 (1987).

[12] P. Mohanty and R.A. Webb, "Anomalous conductance distribution in quasi-one-dimensional gold wires: possible violation of the one-parameter scaling hypothesis," Phys. Rev. Lett. 88, 146601 (2002).

[13] P. Mohanty and R.A. Webb, "High-field measurements of electron decoherence time in metallic nanowires: switching off magnetic impurity spins," Phys. Rev. Lett. 91, 066604 (2003).

[14] S. Feng, in B. L. Altshuler, P.A. Lee, and R. Webb, eds., "Mesoscopic Phenomena in Solids," pages 107-130 (Elsevier, New York, 1991).

[15] N. Feng, in B. L. Altshuler, P.A. Lee, and R. Webb, eds., "Mesoscopic Phenomena in Solids," pages 131- (Elsevier, New York, 1991).

[16] P. Esquinazi, ed., Tunneling Systems in Amorphous and Crystallie Solids (Springer, New York, 1998).

[17] S. Washburn and R.A. Webb, "Quantum transport in small disordered samples from the diffusive to the ballistic regime," Rep. Prog. Phys. 55, 1311 (1992). 
[18] H. Haucke, S. Washburn, A.D. Benoit, C.P. Umbach, and R.A. Webb, "Universal scaling of nonlocal and local resistance fluctuations in small wires," Phys. Rev. B 41, 12454-12462 (1990).

[19] H.U. Baranger, A.D. Stone, and D.P. DiVincenzo, "Resistance fluctuations in multiprobe microstructures: length dependence and nonlocality," Phys. Rev. B 37, 6521-6524 (1988).

[20] G. Tatara and H. Fukuyama, "Resistivity due to a domain wall in ferromagnetic metal," Phys. Rev. Lett. 78, 3773-3776 (1997).

[21] K. Hong and N. Giordano, "Approach to mesoscopic magnetic measurements," Phys. Rev. B 51, 9855-9862 (1995).

[22] V.K. Dugaev, P. Bruno, and J. Barnas, "Weak localization in ferromagnets with spin-orbit interaction," Phys. Rev. B 64, 144423 (2001).

[23] M. Aprili, J. Lesueur, L. Dumoulin, and P. Nédellac, "Weak localization and electron-electron interaction in percolating nickel films," Sol. State Comm. 102, 41-46 (1997).

[24] B.L. Altshuler and B.Z. Spivak, "Variation of the random potential and the conductivity of samples of small dimensions," JETP Lett. 42, 447-450 (1985).

[25] S.C. Feng, P.A. Lee, and A.D. Stone, "Sensitivity of the conductance of a disordered metal to the motion of a single atom - implications for $1 / f$ noise," Phys. Rev. Lett. 56, 1960-1963 (1986).

[26] Y. Lyanda-Geller, I.L. Aleiner, and P.M. Goldbart, "Domain walls and conductivity of mesoscopic ferromagnets," Phys. Rev. Lett. 81, 3215-3218 (1998).

[27] Y. Koyama and Y. Takane, "Quantum interference effect on the conductance of a ferromagnetic wire with a domain wall," J. Phys. Soc. Japan 72, 634-644 (2003).

[28] Y. Takane, "Dephasing due to spin-wave excitations in ferromagnetic metals," J. Phys. Soc. Japan 72, 1155-1160 (2003).

[29] J. Aumentado and V. Chandrasekhar, "Magnetotransport in mesoscopic ferromagnetic particles," Physica B 284-288, 1742-1743 (2000).

[30] S. Kasai, E. Saitoh, and H. Miyajima, "Quantum transport properties in ferromagnetic rings at low temperature," J. Appl. Phys. 93, 8427-8429 (2003).

[31] E. Saitoh, S. Kasai, and H. Miyajima, "Aharonov Bohm oscillation in a multi-domain ferromagnetic $\mathrm{Fe}_{0.10} \mathrm{Ni}_{0.81}$ ring," Physica E 18, 237 (2003).

[32] Y. Imry, Introduction to Mesoscopic Physics (Oxford University, New York, 1997).

[33] F. Pierre and N.O. Birge, "Dephasing by extremely dilute magnetic impurities revealed by Aharonov-Bohm oscillations," Phys. Rev. Lett. 89, 206804 (2002).

[34] A. Trionf, S. Lee, and D. Natelson, "Electronic coherence in metals: comparing weak localization and universal conductance fluctuations," Phys. Rev. B 70, 041304(R) (2004).

[35] A. Trionfi, S. Lee, and D. Natelson, "Time-dependent universal conductance fluctuations and coherence in AuPd and Ag," Phys. Rev. B 71, in press (2005). 
[36] D. Hoadley, P. McConville, and N.O. Birge, "Experimental comparison of the phase-breaking lengths in weak localization and universal conductance fluctuations," Phys. Rev. B 60, 56175625 (1999).

[37] P. McConville and N.O. Birge, "Weak localization, universal conductance fluctuations, and $1 / f$ noise in Ag," Phys. Rev. B 47, 16667-16670 (1993).

[38] Ya. M. Blanter, "Electron-electron scattering rate in disordered mesoscopic systems," Phys. Rev. B 54, 12807-12819 (1996).

[39] I.L. Aleiner and Y.M. Blanter, "Inelastic scattering time for conductance fluctuations," Phys. Rev. B 65, 115317 (2002).

[40] S. Lee, A. Trionf, and D. Natelson, "Quantum coherence in a ferromagnetic metal: timedependent conductance fluctuations," Phys. Rev. B 70, 212407 (2004). 